\title{
Quantification of Stretching in the Ventricular Wall and Corpus Callosum and Corticospinal Tracts in Hydrocephalus before and after Ventriculoperitoneal Shunt Operation
}

\author{
Hans von Holst ${ }^{1,2}$ and Xiaogai $\mathrm{Li}^{2}$ \\ ${ }^{1}$ Department of Neurosurgery, Karolinska University Hospital, 17176 Stockholm, Sweden \\ ${ }^{2}$ Division of Neuronic Engineering, School of Technology and Health, Royal Institute of Technology (KTH), \\ KTH-Flemingsberg Alfred Nobels Allé 10, Huddinge, 14152 Stockholm, Sweden
}

Correspondence should be addressed to Hans von Holst; hans.vonholst@karolinska.se

Received 6 March 2013; Accepted 10 April 2013

Academic Editor: Hang Joon Jo

Copyright (C) $2013 \mathrm{H}$. von Holst and X. Li. This is an open access article distributed under the Creative Commons Attribution License, which permits unrestricted use, distribution, and reproduction in any medium, provided the original work is properly cited.

In this study, we establish a quantitative model to define the stretching of brain tissue, especially in ventricular walls, corpus callosum (CC) and corticospinal (CS) fiber tracts, and to investigate the correlation between stretching and regional cerebral blood flow (rCBF) before and after ventriculoperitoneal shunt operations. A nonlinear image registration method was used to calculate the degree of displacement and stretching of axonal fiber tracts based on the medical images of six hydrocephalus patients. Also, the $\mathrm{rCBF}$ data from the literature was analyzed and correlated with the strain level quantified in the present study. The results showed substantial increased displacement and strain levels in the ventricular walls as well as in the CC and CS fiber tracts on admission. Following shunt operations the displacement as well as the strain levels reduced substantially. A linear correlation was found to exist between strain level and the rCBF. The reduction in postoperative strain levels correlated with the improvement of rCBF. All patients improved clinically except for one patient due to existing dementia. These new quantitative data provide us with new insight into the mechanical cascade of events due to tissue stretching, thereby provide us with more knowledge into understanding of the role of brain tissue and axonal stretching in some of the hydrocephalus clinical symptoms.

\section{Introduction}

Hydrocephalus is the consequence of various causes such as congenital malformations, subarachnoid hemorrhage, traumatic brain injury, and benign and malignant brain tumors. Regardless of etiology the common denominator among the patients is obstruction of cerebrospinal fluid interfering with the brain tissue function. The symptoms and signs are somewhat different depending on whether the patient is an infant, is child, or receives the hydrocephalus in mature age.

The expansion of the ventricular system influences the surrounding areas in the brain tissue in various intensities. The clinical condition is usually defined as the Hakim triad of symptoms including gait apraxia, cognitive disturbance, and incontinence. Initially the white matter suffers more than the grey matter with reversible changes following shunt operation which reduces the expanding ventricles.
Depending on the age at which hydrocephalus develops together with the magnitude of ventricular expansion, the neuropathological pattern is different in severity. When longstanding the mechanical distortion results in more advanced neuropathological signs such as damage to the axons and myelin. Also, when the cerebral arteries and capillaries are substantially distorted, there is a potential of reduced regional cerebral blood flow which may further interfere with the clinical condition $[1,2]$.

Stretching/compression of the ventricular wall [3], the corpus callosum (CC) $[4,5]$ and corticospinal (CS) $[6$, 7] fiber tracts in hydrocephalus patients has been widely recognized in many previous research investigations. Also an early hypothesis suggested that the compression and/or deformation of the CS tracts due to enlargement of the ventricles in hydrocephalus patient may contribute to the gait disturbance [8]. However, there is a lack of study providing 
quantitative data of the axonal stretching level, and how the mechanical stretching may influence the axonal functions remains unclear until now. A quantitative data should also provide us more insight to understand the involvement of axonal fiber stretching in clinical symptoms.

Thus, the aim of the present study was

(i) to analyze the displacement and strain levels in patients with hydrocephalus by using a numerical simulation method focusing on the ventricular walls, CC and CS fiber tracts before and after shunt operations and

(ii) to estimate the regional cerebral blood flow after treatment and the correlation to the strain level after the neurosurgical procedure.

\section{Material and Methods}

2.1. Medical Images. Medical images from computed tomography (CT) and magnetic resonance (MR) imaging of the six patients with hydrocephalus were investigated retrospectively before (Figure 2) and after (Figure 3) a ventriculoperitoneal shunt operation. In this study, only the geometry of the brain is of concern to the strain level quantification. Therefore, both CT and Resonance MR Images are acceptable. This study was approved by the local research ethical committee of Karolinska University Hospital, Stockholm County.

2.2. Image Registration to Obtain Displacement Field. Image registration aims at finding a displacement field between a fixed image and moving image to align both images as accurate as possible [9]. A nonlinear registration method, the Diffeomorphic Demons (DD) algorithm [9] implemented in the open-source software Slicer 3D, was used in this study which has been used to quantify the displacement field occurred during decompressive craniotomy in previous studies $[10$, 11]. The core components of the DD registration algorithm including similarity measures and the regularization model which will be presented.

Given a fixed image $F(\cdot)$ and a moving image $M(\cdot)$, intensity-based image registration is posed as an optimization problem that aims at finding a spatial mapping that will align the moving image to the fixed image. The transformation $s(\cdot): \mathbb{R}^{D} \rightarrow \mathbb{R}^{D}, p \mapsto s(p)$ models the spatial mapping of a particular point $p$ from the fixed image space to the moving image space [12]. The similarity criterion $E_{\text {sim }}(F, M \circ$ $s$ ) measures the quality or the goodness of the matching of a given transformation. In this study, binary images are used, and the sum of the square difference (SSD) suffices for our application as defined as

$$
\begin{aligned}
E_{\text {sim }}(F, M \circ s) & =\frac{1}{2}\|F-M \circ s\|^{2} \\
& =\frac{1}{2 \Omega_{p}} \sum_{p \in \Omega_{p}}|F(p)-M(s(p))|^{2},
\end{aligned}
$$

where $M \circ s$ represents the morphed moving image, $\Omega_{p}$ is the region of overlap between $F$ and $M \circ s$. The problem now becomes an optimization problem to find a transformation $s$ over a given space that minimizes the similarity energy function $E_{\text {sim }}$.

In order to end up with a global minimization of a well-posed criterion, the similarity energy function in (1) was modified by introducing an auxiliary variable (i.e., correspondence $c$ ) in the registration process [13]. The introduction of this auxiliary variable $c$ decouples the complex minimization into simple and efficient steps by alternating optimization over $c$ and $s$. Considering a Gaussian noise on the displacement field, the global energy then becomes [12]

$$
\begin{aligned}
E(c, s)= & \frac{1}{\sigma_{i}^{2}} E_{\text {sim }}(F, M \circ s)+\frac{1}{\sigma_{x}^{2}} \operatorname{dist}(s, c)^{2} \\
& +\frac{1}{\sigma_{T}^{2}} E_{\text {reg }}(s),
\end{aligned}
$$

where $\sigma_{i}$ accounts for the noise on the image intensity, $\sigma_{x}$ accounts for a spatial uncertainty of the correspondences, and $\sigma_{T}$ controls the amount of regularization that is needed: $\operatorname{dist}(s, c)=\|c-s\|$ and $E_{\text {reg }}(s)=\|\nabla s\|$.

The model assumes that so-called "demons" at every voxel from the fixed image are applying forces that push the voxels of the moving image to match up with the fixed image. The transformation $s$ is driven by a "demons force" derived from the assumption of image intensity conservation [14]. The original demons algorithm has been modified and allows retrieving small and large dense displacement field defined as [15]

$$
\mathbf{U}(p)=\frac{F(p)-M(s(p))}{\|\nabla F(p)\|^{2}+\alpha^{2}(F(p)-M(s(p)))^{2}} \nabla F(p),
$$

where $\mathbf{U}(p)$ is the displacement at point $p$ defined in the fixed image, $\alpha$ is a positive homogenization factor. The updated unconstrained dense displacement field is computed based on an optical flow computation at each voxel at every iteration. The resulting updated field is added to the global deformation field, and the displacement field is regularized by applying a Gaussian smoothing filter.

In order to solve the displacement field $\mathbf{U}$, the global energy defined in (2) needs to be minimized. This energy function allows the whole optimization procedure to be decoupled into two simple steps. The first step solves for the correspondence $c$ by optimizing $\left(1 / \sigma_{i}^{2}\right) E_{\text {sim }}+\left(1 / \sigma_{x}^{2}\right)\|s-c\|^{2}$ with respect to $c$ and with $s$ given. The second step solves for the regularization by optimizing $\left(1 / \sigma_{x}^{2}\right)\|s-c\|^{2}+$ $\left(1 / \sigma_{T}^{2}\right) E_{\text {reg }}(s)$ with respect to $s$ and $c$ given [9].

The transformation $s$ in the original Demons method [14] is not constrained and does not provide diffeomorphic transformations. A diffeomorphic extension to the demons framework was proposed by adapting the optimization procedure to a space of diffeomorphic transformations. It is performed by using an intrinsic update step which computes the vector field exponentials of the Lie group of diffeomorphims (see $[9,12]$ for details).

2.3. Strain Level Quantification. From the diffeomorphic Demons registration, the transformation $s$, corresponding 
to a displacement field $\mathbf{U}(\mathbf{X})$, is obtained from (3). The displacement field is defined on every voxel in the fixed image and morphs the fixed image to the moving image. The strain tensor could then be calculated based on the theory of continuum mechanics [16]. A Lagrangian reference frame was assumed at the fixed image space:

$$
s: \mathbf{X}=\mathbf{X}+\mathbf{U}(\mathbf{X}) \text {. }
$$

The displacement field $\mathbf{U}(\mathbf{X})=\left[U_{X}, U_{Y}, U_{Z}\right]$ for each voxel, obtained from the image registration, gives a reasonable alignment to bring the fixed image at point $p$ with coordinates $\mathbf{X}(X, Y, Z)$ to its corresponding point in the moving image at coordinates $\mathbf{x}(x, y, z)$ [9]. The displacement gradient is defined as [16]

$$
\operatorname{grad}(\mathbf{U})=\frac{d \mathbf{U}}{d \mathbf{X}}=\left(\begin{array}{ccc}
\frac{d U_{X}}{d X} & \frac{d U_{X}}{d Y} & \frac{d U_{X}}{d Z} \\
\frac{d U_{Y}}{d X} & \frac{d U_{Y}}{d Y} & \frac{d U_{Y}}{d Z} \\
\frac{d U_{Z}}{d X} & \frac{d U_{Z}}{d Y} & \frac{d U_{Z}}{d Z}
\end{array}\right)
$$

The deformation gradient is defined as

$$
\mathbf{F}=\frac{d \mathbf{x}}{d \mathbf{X}}=\frac{d(\mathbf{X}+\mathbf{U}(\mathbf{X}))}{d \mathbf{X}}=\mathbf{I}+\frac{d \mathbf{U}}{d \mathbf{X}}=\mathbf{I}+\operatorname{grad}(\mathbf{U}) \text {. }
$$

The Lagrangian finite strain tensor $\mathbf{E}$ is defined as

$$
\mathbf{E}=\frac{1}{2}\left(\mathbf{F}^{\mathrm{T}} \mathbf{F}-\mathbf{I}\right) \text {. }
$$

The Lagrangian finite strain tensor, $\mathbf{E}$, is a $3 \times 3$ symmetric tensor representing the deformation of a point. Different scalar indices that can be derived from the tensors, especially the principal strains which represent the maximum and minimum normal strains experienced at a point, are characterized by the tensor eigenvalues.

Strain level represents the deformation of brain tissue due to the occurrence of a disease. In order to quantify the brain tissue deformation, a dense displacement field representing the brain tissue motion from healthy stage to hydrocephalus stage is needed. For this, the main premise is the images acquired at two distinct states: at healthy state and at diseased state. Since the brain images of the patient under healthy state were not available, we proposed a strategy to recover a healthy brain for a specific patient by combing the information from the diseased brain geometry together with the brain atlas by a nonlinear image registration method [10].

The lateral ventricles and brain tissue were firstly manually segmented from the recovered healthy brain and hydrocephalic brain images. A rigid registration step was first used to centre the images about the same point which were the input volumes to nonlinear registration process. In principle, the segmented healthy brain should be chosen as the fixed image so as to capture the motion of brain tissue from the healthy to hydrocephalic state. In this study, since the registration performs better from healthy brain to the hydrocephalic brain image, we chose the hydrocephalic brain image as the fixed image, and the obtained displacement field was then inverted by an itkIterativeInverseDisplacementFieldImageFilter algorithm implemented in ITK (see the description for the approach on ITK's website http://www.itk.org). The final obtained displacement for further processing is the inverted displacement field defined on the recovered healthy brain space which represents the structural brain change that occurred from the healthy to hydrocephalus state.

From the obtained displacement field, a quantitative description of the nerve tissue deformation that occurred during hydrocephalus development can be derived in the form of the finite Lagrange strain tensor [16, 17]. Different scalar indices can be derived from the strain tensor, especially, the 1st principal strain, defined as the maximum eigenvalue of Lagrange strain tensor $\mathbf{E}$, representing the maximum stretching was used to describe the stretching of brain tissue.

\subsection{Axonal Fiber Tract Extraction and Healthy Brain Shape} from Atlas. From a series of diffusion weighted (DW) images, the effective diffusion tensor, $\mathbf{D}$, can be estimated using the relationship between the measured signal intensity at each voxel and the applied magnetic field gradient sequence [18, 19]. The diffusion tensor is symmetric (i.e., $D_{i j}=D_{j i}$ ), and thus it contains only six unique values. Given this, at least six noncollinear diffusion gradient directions are required to determine the diffusion tensor [20,21].

Once the diffusion tensor $\mathbf{D}$ is determined, different indices can be derived to provide information for each voxel in the image. The diffusion tensor is a real, symmetric secondorder tensor. Mathematically, this entails a linear rotation of the diffusion tensor to diagonalize it thereby setting offdiagonal elements to zero [22]:

$$
\begin{aligned}
\mathbf{D} & =\left[\begin{array}{lll}
D_{x x} & D_{x y} & D_{x z} \\
D_{y x} & D_{y y} & D_{y z} \\
D_{z x} & D_{z y} & D_{z z}
\end{array}\right] \\
& =\left[\mathbf{e}_{1} \mathbf{e}_{1} \mathbf{e}_{3}\right]\left[\begin{array}{ccc}
\lambda_{1} & 0 & 0 \\
0 & \lambda_{2} & 0 \\
0 & 0 & \lambda_{3}
\end{array}\right]\left[\mathbf{e}_{1} \mathbf{e}_{1} \mathbf{e}_{3}\right]^{T},
\end{aligned}
$$

where $\lambda_{1}, \lambda_{2}$, and $\lambda_{3}$ are eigenvalues $\left(\lambda_{1}>\lambda_{2}>\lambda_{3}\right)$, and the corresponding eigenvectors are $\mathbf{e}_{1}, \mathbf{e}_{2}, \mathbf{e}_{3}$. The eigenvalues of the diffusion tensor provide diffusion coefficients along the orientations defined by its respective eigenvectors [22]. $\lambda_{1}$ represents the greatest diffusion value along a fiber axis, denoted by the direction vector $\mathbf{e}_{1} . \lambda_{2}, \lambda_{3}$ represent the diffusion value along two axes perpendicular to $\mathbf{e}_{1}$. Because $\mathbf{D}$ is symmetric and positive definite, its three eigenvectors (principal coordinate directions) $\mathbf{e}_{1}, \mathbf{e}_{2}$, and $\mathbf{e}_{3}$ are orthogonal [23].

The Fractional Anisotropy (FA) is the most commonly used anisotropy measure and is a normalized expression of the tensor eigenvalues [24] according to

$$
\mathrm{FA}=\sqrt{\frac{3\left(\left(\lambda_{1}-\bar{\lambda}\right)^{2}+\left(\lambda_{2}-\bar{\lambda}\right)^{2}+\left(\lambda_{3}-\bar{\lambda}\right)^{2}\right)}{2\left(\lambda_{1}^{2}+\lambda_{2}^{2}+\lambda_{3}^{2}\right)}},
$$

where $\bar{\lambda}$ is the mean of the eigenvalues of the diffusion tensor. 
TABLE 1: Summary of six hydrocephalic patients including age, clinical symptoms before treatment with a ventriculoperitoneal shunt postoperative image performance and outcome evaluated in days after the operation.

\begin{tabular}{lcccc}
\hline Patient/age & Gait disturbance & Cognitive deficit & $\begin{array}{c}\text { Clinical symptoms } \\
\text { Incontinence }\end{array}$ & Improved outcome/days after treatment \\
\hline KP/45 & Yes & Yes & No & Yes/63 \\
KA/47 & Yes & Yes & No & Yes/96 \\
KB/72 & Yes & Yes & Yes & Yes/30 \\
KF/70 & No & Yes & Yes & Yes/27 \\
PN/57 & No & Yes & No & Yes/19 \\
SB/71 & Yes & Yes & No & No/21 \\
\hline
\end{tabular}

Once the fractional anisotropy (FA) and the preferred diffusion direction have been created, it is possible to perform fiber tracking or diffusion tensor tractography. Streamline tractography is one of the methods for fiber tract estimation $[25,26]$. Streamline tractography uses the maximum orientation described by the eigenvector $\mathbf{e}_{1}$ associated with the largest eigenvalue $\lambda_{1}$, as an estimate of local tract orientation. It assumes that $\lambda_{1}$ associated with the maximum eigenvector is the main fiber direction.

In this study, white matter fiber tracts were extracted from ICBM DTI-81 atlas provided by the international consortium for brain mapping (ICBM) [27]. The T2-weighted image from the same dataset which represents an average adult brain was used for recovery of a healthy brain as described in a previous section. The atlas is based on probabilistic tensor maps obtained from 81 normal adults ranging from 18 to 59 years of age. From the diffusion tensor imaging, the Fractional Anisotropy (FA) could be calculated which is a scalar value representing the anisotropic property. The colorcoded FA value calculated from the DTI tensor is displayed in (Figure 1, left) where the color reflects the white matter orientation (red (right-left), green (anterior-posterior), and blue (superior-inferior)). The fiber tracts which pass through the predefined region of interests (ROI) are CC and CS fiber tracts. The corresponding sagittal and coronal sections of the T2-weighted images from the same dataset are shown in Figure 1 upper row on the right.

Streamline method $[26,28]$ was used to extract white matter fiber tracts. The final extracted white matter tracts in the whole brain contain the polylines with a corresponding diffusion tensor at each fiber point (Figure 1, lower row: left and middle), from which the CC and CS fiber tracts were isolated (Figure 1, lower row: right). The obtained fiber tracts from the atlas were then adapted to the patient brain by applying the obtained displacement field which represent the cranial structural shape difference between the patient and atlas. This gave the fiber tracts for the recovered healthy brain for a specific patient.

\section{Results}

3.1. Patient Information. The average age of the six patients with hydrocephalus was calculated to 60 years. Clinical symptoms among the six patients showed gait disturbances in four cases and cognitive deficits in all patients while only two had urinary incontinence (Table 1). Five patients improved in their clinical symptoms, individually evaluated between 19 and 96 days after the shunt operation, while the sixth patient did not probably due to the presence of dementia.

3.2. Strain Level at Ventricular Wall, CC and CS Fiber Tracts. The increased strain levels in the six patients are presented for preoperative (Figure 2) and postoperative stages (Figure 3). Before and after the operation all patients had a substantial dilatation of the lateral ventricles in various degrees although in less degree after the treatment. The ventricular dilatation caused increased strain levels not only in the ventricular walls ranging from $99 \%$ to $333 \%$, but also in the brain tissue surrounding the ventricles in different degrees. As a consequence, the increased strain levels found in the CC fiber tracts ranged from $58 \%$ to $139 \%$ and in the CS fiber tracts from $29 \%$ to $74 \%$ among the patients (Figure 7 ).

After the ventriculoperitoneal shunt had been installed, the lateral ventricles decreased although not to an extent as could be expected after such an operation. Thus, the postoperative strain levels of the ventricular walls decreased in all six patients ranging from $9 \%$ to $60 \%$ based on the preoperative values (Figure 7).

In a similar way, although decreased after the shunt operation, the strain levels in the CC and CS fiber tracts were still on a surprisingly high level ranging from $9 \%$ to $56 \%$ for CC fiber tracts and from $5 \%$ to $20 \%$ in the CS fiber tracts (Figure 7). The strain level data showed normal distribution, and therefore, a paired sample $t$-test was used to compare the CC and CS fiber strain levels. The results were evaluated by a 1-tailed $t$-test at $95 \%$ confidence level. It was found that the strain levels in CC fiber tracts are significantly higher than those of CS fiber tracts for both pre- $(P<0.05)$ and postcraniotomy period $(P<0.05)$. This indicates a more stretched scenario for the CC fiber tracts.

The trend was that those patients with the highest ventricular dilatation and increased strain levels also showed the highest strain levels in the CC and CS fiber tracts both before and after treatment. A similar trend was found between CC and CS fiber tracts. Also, since the CC fiber tracts are closer to the expanded ventricles, it seems logical that the increased strain levels are higher in these fibers compared to those found in the CS fiber tracts which are anatomically located further away from the ventricles. 


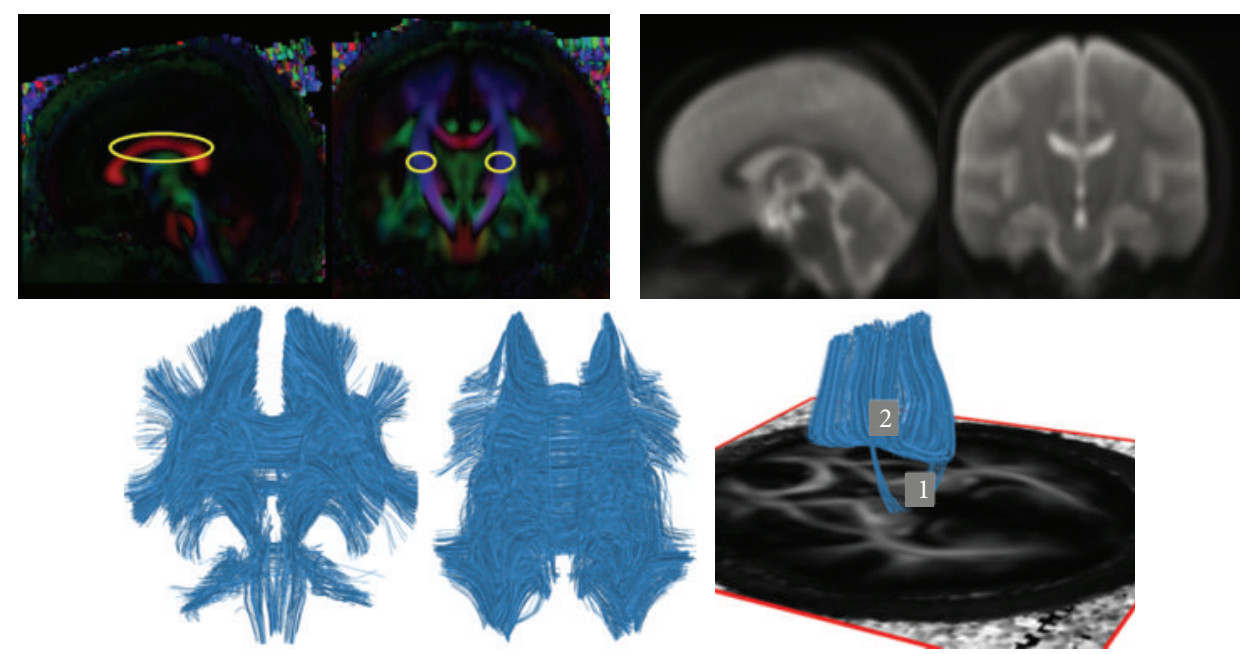

FIGURE 1: Upper row: color-coded FA map calculated from the ICBM DTI-81 atlas (left). Sagittal and coronal sections of T2-weighted images of the atlas (right). Lower row: extracted fiber tracts from the ICBM DTI-81 atlas in the whole brain. Rear view (left) and top view of the extracted fiber tracts in the whole brain (middle). Note the isolated CC and CS tracts (right).

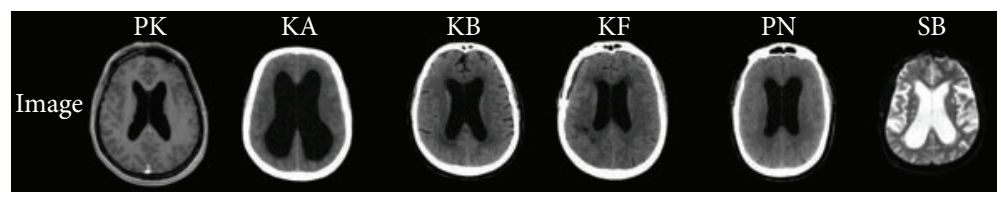

(a)

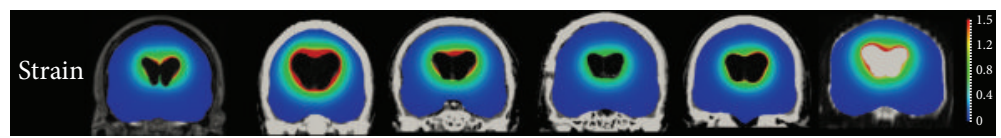

(b)

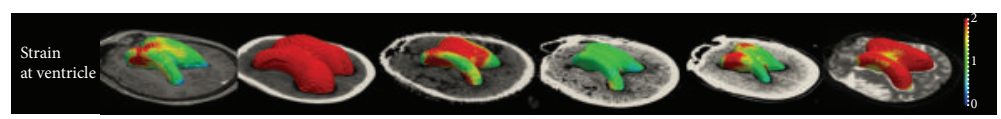

(c)

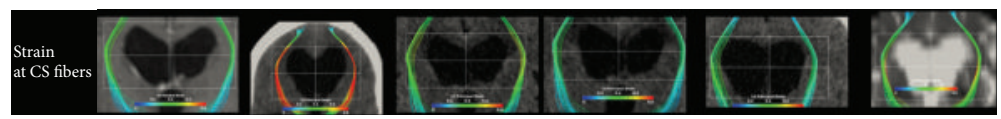

(d)

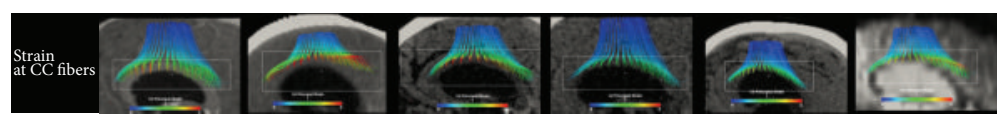

(e)

Figure 2: Overview of the 6 patients at their preoperative stages. Each row from top to bottom represents the medical images used for nonlinear image registration from which the strain level was quantified in the entire brain, and the calculated strain levels are illustrated at coronal sections and the ventricular walls. At bottom the strain level at CS and CC fiber tracts is presented.

The individual follow-up time ranged from 19 to 96 days. The increased strain levels should be expected to decrease more in those patients with longer follow-up times compared to those with a short follow-up time. However, there was no such pattern found among the patients in this study.

The result is exemplified with one of the six patients (patient PK) which is presented in more detail (Figure 4). As defined previously, the dilatation of the lateral ventricles causes distortion of most of the surrounding axonal fiber tracts and where the CC and CS fiber tracts are addressed here. Thus, during the hydrocephalus development the CS fiber tracts were displaced outwards by the arrows representing the displacement vector obtained from image registration (Figure 4, upper row). The maximum displacement evaluated was $10.7 \mathrm{~mm}$ (Figure 4, upper row: left). The closer to the lateral ventricles, the larger the displacement found in the CS 


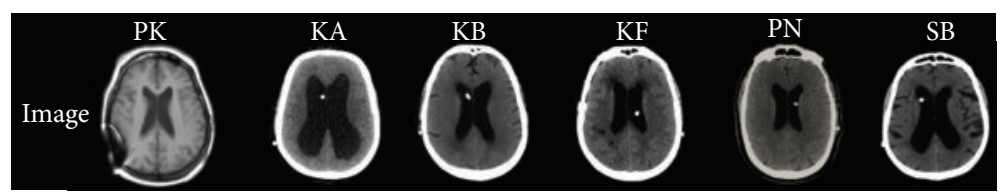

(a)

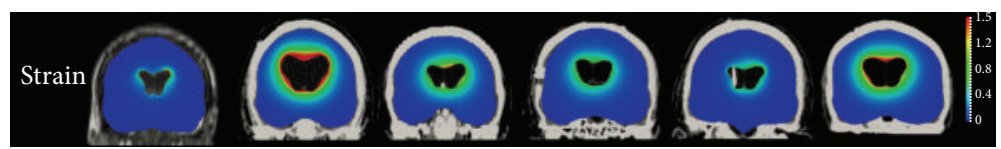

(b)

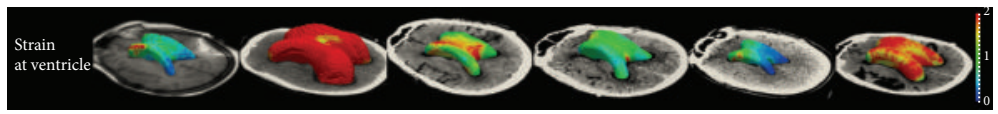

(c)

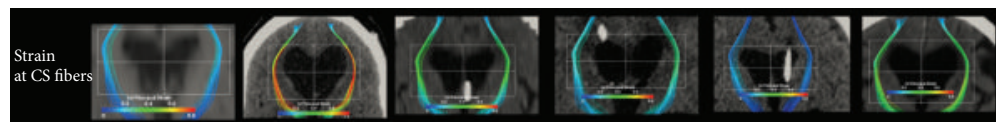

(d)

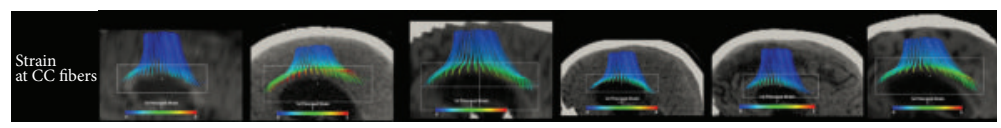

(e)

FiguRE 3: Overview of the 6 patients at their postoperative stages. Each row from top to bottom represents the medical images used for nonlinear image registration from which the strain level was quantified in the entire brain, and the calculated strain levels are illustrated at coronal sections and the ventricular walls. At bottom the strain level at CS and CC fiber tracts is presented.
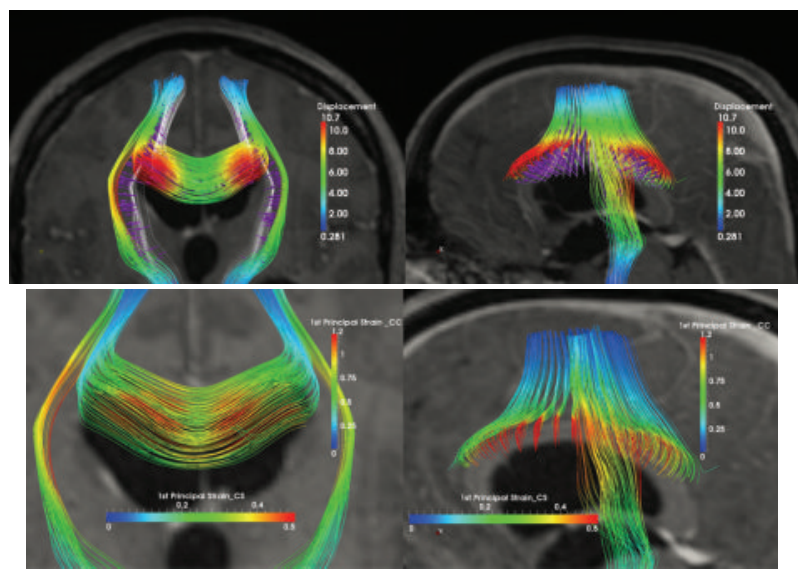

Figure 4: Upperrow: displacement of the CC and CS fiber tracts during hydrocephalus development overplayed with the preoperative brain image at coronal cross-section (left) and sagittal cross-section (right). The axonal tracts with white color represent the tracts at the healthy stage, the purple arrow shows the displacement occurred during hydrocephalus development with the arrow length in proportion to the displacement magnitude, and the colored fiber tracts represent the distorted tracts at postoperative stage with color scale legend indicating magnitude of displacement. Lower row: the 1st principal strain levels at the CS and the CC fiber tracts. Note that the color scale for the CS and CC fiber tracts is different in order to have a better illustration. fiber tracts. This resulted into compression of the CS fiber tracts close to the lateral ventricles. For axonal CC fiber tracts, the displacement field showed a more dispersed pattern with a maximum value up to $15.4 \mathrm{~mm}$ located at the anterior horn of lateral ventricle (Figure 4, upper row: right).

The strain levels for both CS and CC fiber tracts (Figure 4, lower row) showed that the CS fiber tracts presented a lower value compared to that found in the CC fiber tracts. Thus, the maximum strain level increase in the CS fiber tracts was 0.5 , or $50 \%$, while that in the CC fiber tracts was as high as 1.2 or $120 \%$.

When comparing the average strain levels found in the ventricles, the CC and CS fibers, the trend seemed clear that the longer distance from the ventricles the less the strain levels both before and after the neurosurgical procedure (Figure 7). Hence, the difference was the least in fiber tracts in the periphery of the brain tissue for both pre-and postoperative stages.

To evaluate the strain level at brain tissue with further distance from the lateral ventricles, the original ventricular walls were scaled outwards until it reached the cranial boundary and which was taken as the maximum distance for evaluation. Between the original normal ventricles and the maximum dilated ventricles, ten different-sized lateral ventricles were created by equally interpolating the two extreme cases. The obtained different-sized ventricles are 

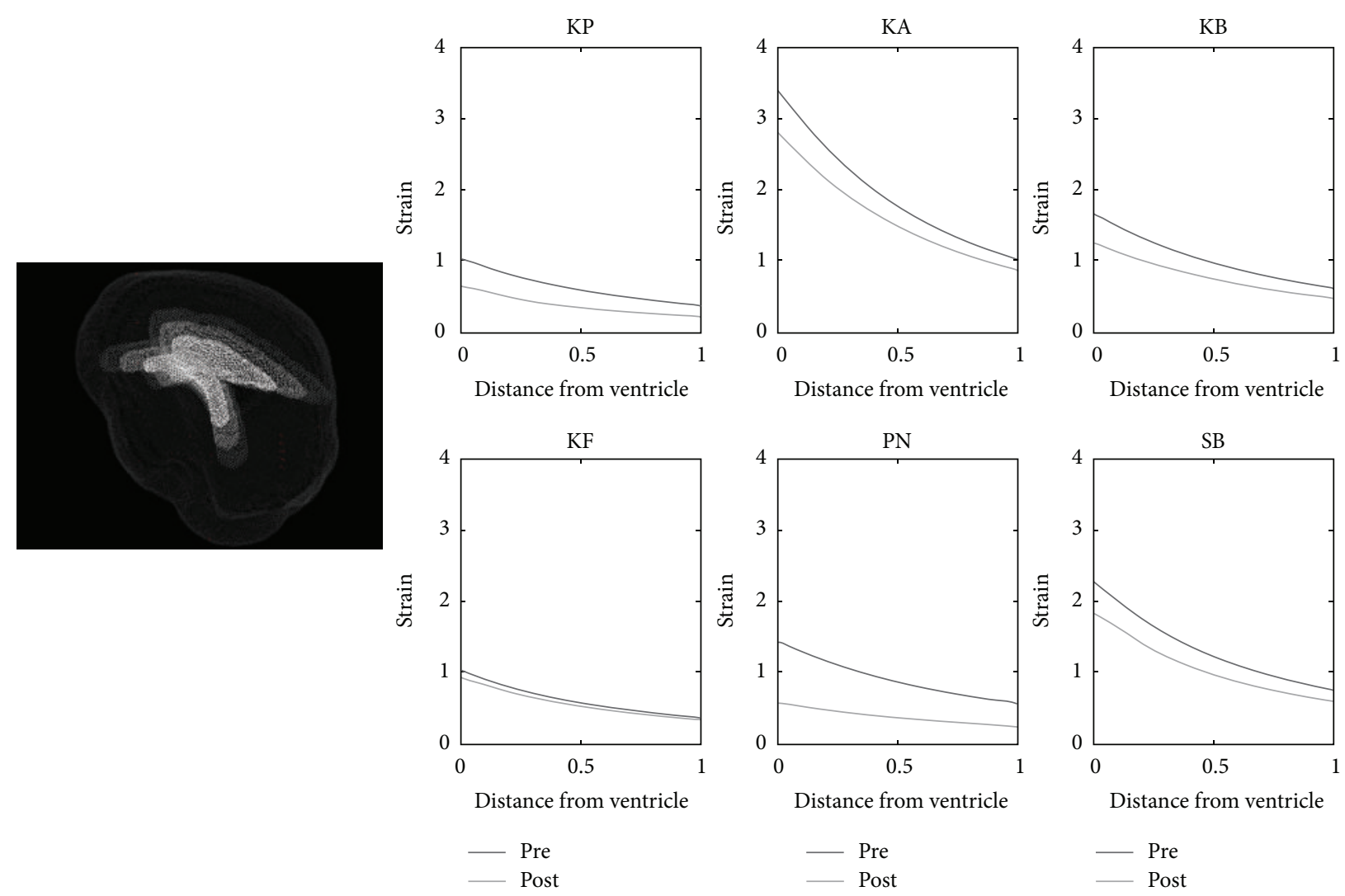

Figure 5: Relationship between the distance from the lateral ventricles and the mean strain level of brain tissue. The picture on the left illustrates the interpolated different-sized ventricles which were used for average strain level calculation at different distances. A decreased strain level was found after the neurosurgical procedure in all of the 6 patients in various degrees.

illustrated in Figure 5, left with only three ventricles shown aiming at a better illustration. The obtained, interpolated ventricles were then used to resample the strain levels in the entire brain and based on which the average strain levels at different distances to the ventricles were calculated. As the brain sizes are different across the patients in the present study, the distance from the ventricles was normalized for each patient by dividing the brain size to the largest distance in that particular patient. This yielded distances range from 0 , that is, brain tissue adjacent to the ventricles, to 1 , that is, the outermost brain tissue, which were binned in an increment of 0.1 . A profile of the strain levels in brain tissue as a function distance from the ventricles could then be plotted. Thus, the relationship between the distance from the ventricles and the average strain levels showed a logarithmic profile in all patients both at the pre- and postoperative periods (Figure 5). The evaluation showed decreasing strain levels with increasing distances from the ventricles for each patient although in different degrees.

An earlier study has shown a logarithmic correlation between the regional cerebral blood flow (rCBF) and the distance from the lateral ventricles in hydrocephalic patients [1]. Thus, the displacement and increased strain levels presented in this study may have a substantial impact on the rCBF among these patients. By combining the rCBF values from that earlier study at a distance from the lateral ventricles together with the increased strain levels found in this study at the same distance, the relationship between CBF and strain levels was plotted (Figure 6) showing a linear correlation between the increased strain level in this study and the rCBF $(r=0.9905, P<0.0001)$ from the earlier study [1]. Thus, as illustrated at one point in the figure, by reducing the increased strain level from preoperative $176 \%$ to postoperative $130 \%$ shown on the horizontal axis, the $\mathrm{rCBF}$ increased from about 6.5 to $11.4 \mathrm{~mL} / 100 \mathrm{mg} / \mathrm{min}(\mathrm{rCBF}$ in Figure 6$)$.

\section{Discussions}

All six patients had increased displacement as well as profound increased strain levels in the surrounding brain tissue both before and, although less pronounced, after the neurosurgical procedure. Also, all patients were defined with cognitive disturbances. Incontinence was defined in four of the patients and without any correlation to displacement or strain level. However, the neurosurgical procedure resulted in a definite reduction of both displacement and strain level. In those two cases with the lowest increase of displacement and strain level there was no gait ataxia. This study, to the best of our knowledge, is the first to provide a quantitative view regarding the stretching of ventricular wall, CC and CS tracts in hydrocephalic patients. Further, a close correlation was found to exist between the increased strain level and the rCBF. 


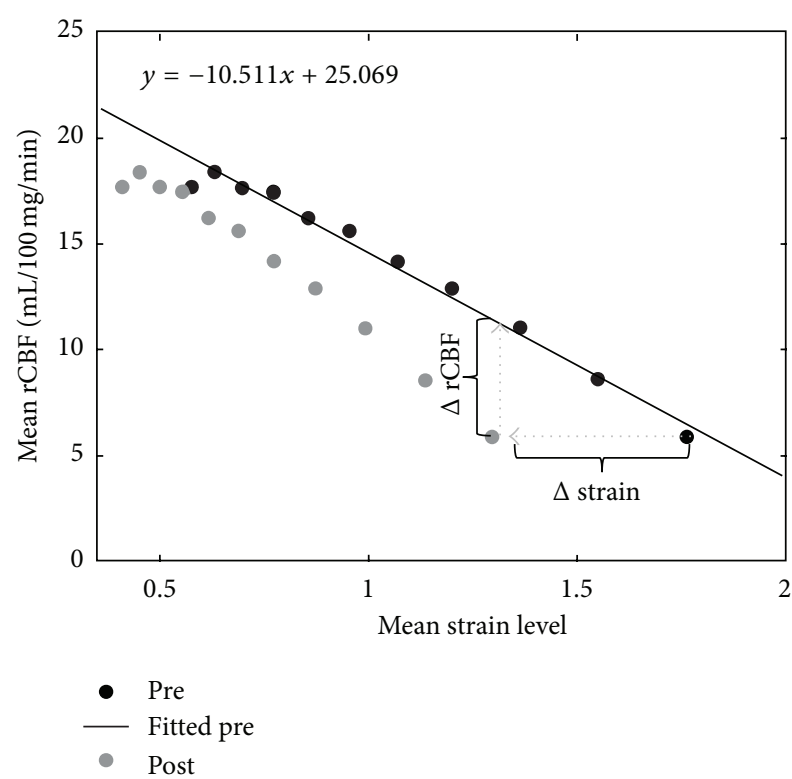

FIgURE 6: A linear relationship $(r=0.9905, P<0.0001)$ was found to exist between the preoperative strain levels ( $x$-axis where $1=100 \%$ strain level increase) and the regional cerebral blood flow ( $\mathrm{rCBF}$ ) (black dots, data from a previous publication [1]. One point was illustrated to show improved $\mathrm{rCBF}$ due to shunt operation (light dots, $\triangle \mathrm{rCBF})$.

The presented results provide direct evidence that Hakim triad could to some extent be explained by the increased strain levels.

Those patients with the largest expansion of the ventricles showed the most profound strain level increase in the fiber tracts of CC and CS, while those with smaller ventricular increase presented a more modest strain level increase. This may indicate that patients with larger ventricles should be expected to present a worse outcome. However, this was not the case in this study. Instead, it is suggested that larger displacement of the ventricles will influence the surrounding brain tissue more distant compared to those patients with less expanded ventricles. Also, the larger the ventricle expansion the more severe the strain level in CC fibers tracts, and this could tentatively interfere negatively with connections between the two hemispheres thereby prolonging the clinical improvement for such patients. Since the CS fiber tracts are anatomically located further away from the ventricles, they had a less pronounced strain level increase which was to be expected.

Experimental studies of axonal stretching have shown a clear correlation between the strain level and axonal function. An increased strain level of 5\% was found to alter neuronal function, while $10 \%$ may cause cell death in rapid axonal stretching models [29]. Bain and Meaney (2000) demonstrated in an animal model that a strain level of approximately $21 \%$ will elicit electrophysiological changes, while a strain of approximately $34 \%$ will cause morphological signs of damage to the white matter [30]. Under slow loading rates, however, axons could tolerate much higher strain levels and with stretch of up to twice of their original length (a strain of 100\%) with no evidence of damage [31]. Using a model of sciatic nerve stretch, Fowler et al. reported that even minimal tension, if maintained for a significant amount of time, may result in loss of neuronal function [32]. Based on these, it should be expected that an increased strain level in both CC and CS fiber tracts in hydrocephalus patients found in the present study very well interferes with the normal conductivity in the axons, hence also interferes with the patients' rehabilitation. When the strain level increase reaches a critical point or threshold, it may very well initiate a biochemical response which may further alter the CC and CS fiber tracts. From a clinical aspect, the sustained increased displacement and increased strain levels of axons may also result in gait disturbances due to the potential interference with the long corticospinal fiber tracts connecting motor and sensory areas with the spinal cord as well as those in the corpus callosum connecting the electrophysiological functions of both hemispheres with each other [33].

The displacement and strain level in hydrocephalus patients belong to a static or semistatic increase thereby allowing the fiber tracts to accept the changes during a longer period. This may favor the rehabilitation even if the increased strain level is substantial due to the very low deformation rate [31]. However, of special interest are elderly people who already have an atrophied brain tissue and which may suffer more than those in employment age. Many of the hydrocephalus patients improve after ventriculoperitoneal shunt treatment. However, there is no treatment for those, who do not improve due to a lack of explanation of the causes. It was suggested that the increased strain levels may cause a disturbance in the electrophysiological function in these fiber tracts, and consequently, a tailored matrix of conductive, organic bioelectrodes could potentially be implanted in the area of corpus callosum fiber tracts thereby counterbalancing the electrophysiological dysfunction as proposed in a previous study [33].

In the present study all patients had indeed a reduction in the strain level postoperatively. However, the reduced strain level was still on a surprisingly high level and could therefore have a negative influence on the anatomy and histology of the nervous tissue together with a sustained alteration in the conductivity of the axons in the white matter. It seems that the reduction of rCBF clearly correlated with that of increased strain levels. Even a small reduction in $\mathrm{rCBF}$ may influence the rehabilitation especially among the vulnerable elderly patients and which should be taken into consideration.

\section{Conclusions}

A numerical method based on nonlinear image registration was used to quantify the stretching of ventricular wall, corpus callosum, and corticospinal axonal fiber tracts in six patients with hydrocephalus both before and after shunt operation. These data provide new insight into the mechanical cascade of events due to tissue stretching, thereby to provide us with more knowledge into understanding of the role of brain tissue and axonal stretching in some of the hydrocephalus clinical symptoms. Moreover, a linear correlation was found 


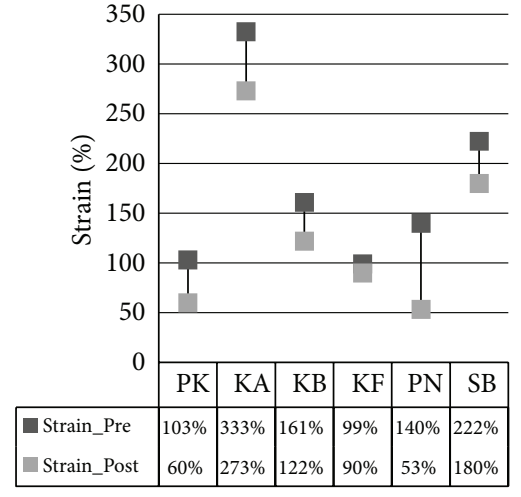

(a)

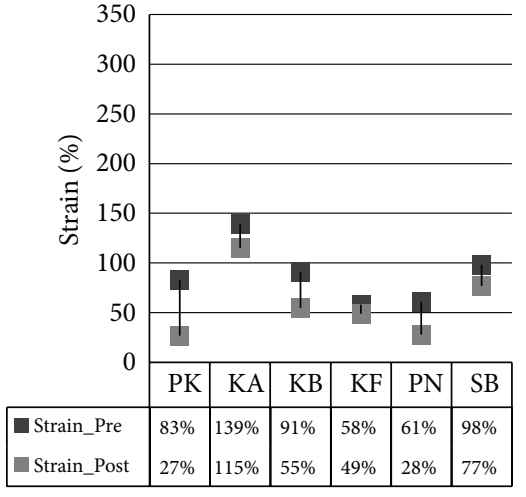

(b)

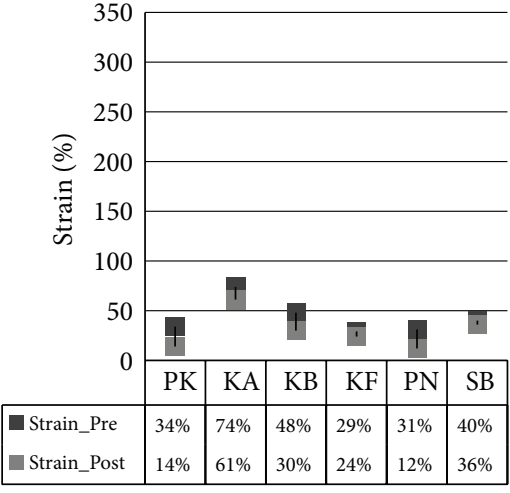

(c)

Figure 7: Diagram of average strain levels at the lateral ventricle (a) CC fiber (b) and CS fiber (c) for all the 6 patients. The horizontal axis is the patient's identification (id), and the vertical axis shows the strain levels for the corresponding patient at precraniotomy stage (Strain_Pre with darker gray color) and postcraniotomy stage (Strain_Post, with lighter gray color). The exact strain level values are presented in the lower table.

to exist between strain level and the rCBF. The combination of increased displacement of the ventricular walls, sustained increased strain levels, and derangement in electrophysiology activity in the white matter together with a reduced $\mathrm{rCBF}$ may, under certain circumstances, has a profound impact on the outcome following hydrocephalus.

\section{Conflict of Interests}

The authors declare that they have no conflict of interests.

\section{Acknowledgment}

The present study was supported by research funds from the Royal Institute of Technology, Stockholm, Sweden.

\section{References}

[1] S. Momjian, B. K. Owler, Z. Czosnyka, M. Czosnyka, A. Pena, and J. D. Pickard, "Pattern of white matter regional cerebral blood flow and autoregulation in normal pressure hydrocephalus," Brain, vol. 127, no. 5, pp. 965-972, 2004.

[2] B. K. Owler and J. D. Pickard, "Normal pressure hydrocephalus and cerebral blood flow: a review," Acta Neurologica Scandinavica, vol. 104, no. 6, pp. 325-342, 2001.

[3] J. M. Miller and J. P. McAllister, "Reduction of astrogliosis and microgliosis by cerebrospinal fluid shunting in experimental hydrocephalus," Cerebrospinal Fluid Research, vol. 4, article 5, 2007.

[4] M. Mataró, M. Matarín, M. A. Poca et al., "Functional and magnetic resonance imaging correlates of corpus callosum in normal pressure hydrocephalus before and after shunting," Journal of Neurology, Neurosurgery and Psychiatry, vol. 78, no. 4, pp. 395-398, 2007.

[5] S. Röricht, B. U. Meyer, C. Woiciechowsky, and R. Lehmann, "Callosal and corticospinal tract function in patients with hydrocephalus: a morphometric and transcranial magnetic stimulation study," Journal of Neurology, vol. 245, no. 5, pp. 280288, 1998.
[6] E. Hattingen, A. Jurcoane, J. Melber et al., "Diffusion tensor imaging in patients with adult chronic idiopathic hydrocephalus," Neurosurgery, vol. 66, no. 5, pp. 917-924, 2010.

[7] T. Hattori, T. Yuasa, S. Aoki et al., "Altered microstructure in corticospinal tract in idiopathic normal pressure hydrocephalus: comparison with Alzheimer disease and Parkinson disease with dementia," The American Journal of Neuroradiology, vol. 32, no. 9, pp. 1681-1687, 2011.

[8] A. Marmarou, M. Bergsneider, P. Klinge, N. Relkin, and P. M. L. Black, "INPH guidelines, part III: the value of supplemental prognostic tests for the preoperative assessment of idiopathic normal-pressure hydrocephalus," Neurosurgery, vol. 57, no. 3, p. S2, 2005.

[9] T. Vercauteren, X. Pennec, A. Perchant, and N. Ayache, "Diffeomorphic demons: efficient non-parametric image registration," NeuroImage, vol. 45, no. 1, pp. S61-S72, 2009.

[10] H. von Holst, X. Li, and S. Kleiven, "Increased strain levels and water content in brain tissue after decompressive craniotomy," Acta Neurochirurgica, vol. 154, no. 9, pp. 1583-1593, 2012.

[11] X. Li, Finite Element and Neuroimaging Techniques to Improve Decision-Making in Clinical Neuroscience, Royal Institute of Technology (KTH), 2012.

[12] T. Vercauteren, X. Pennec, E. Malis, A. Perchant, and N. Ayache, "Insight into efficient image registration techniques and the demons algorithm," in Information Processing in Medical Imaging, pp. 495-506, Springer, New York, NY, USA, 2007.

[13] P. Cachier, E. Bardinet, D. Dormont, X. Pennec, and N. Ayache, "Iconic feature based nonrigid registration: the PASHA algorithm," Computer Vision and Image Understanding, vol. 89, no. 2-3, pp. 272-298, 2003.

[14] J. P. Thirion, "Image matching as a diffusion process: an analogy with Maxwell's demons," Medical Image Analysis, vol. 2, no. 3, pp. 243-260, 1998.

[15] P. Cachier, X. Pennec, and N. Ayache, Fast Non Rigid Matching by Gradient Descent: Study and Improvements of the "Demons" Algorithm, Rapport de Recherche-Institut National de Recherche en Informatique et en Automatique, 1999.

[16] G. A. Holzapfel, Nonlinear Solid Mechanics: A Continuum Approach for Engineering, John Wiley \& Sons, West Sussex, UK, 2000. 
[17] J. Gee, T. Sundaram, I. Hasegawa, H. Uematsu, and H. Hatabu, "Characterization of regional pulmonary mechanics from serial magnetic resonance imaging data," Academic Radiology, vol. 10, no. 10, pp. 1147-1152, 2003.

[18] P. J. Basser and D. K. Jones, "Diffusion-tensor MRI: theory, experimental design and data analysis-a technical review," NMR in Biomedicine, vol. 15, no. 7-8, pp. 456-467, 2002.

[19] P. J. Basser, J. Mattiello, and D. Lebihan, "Estimation of the effective self-diffusion tensor from the NMR spin echo," Journal of Magnetic Resonance B, vol. 103, no. 3, pp. 247-254, 1994.

[20] T. L. Chenevert, "Principles of diffusion-weighted imaging (DW-MRI) as applied to body imaging," in Diffusion-Weighted MR Imaging, pp. 3-17, 2010.

[21] P. J. Basser and C. Pierpaoli, "A simplified method to measure the diffusion tensor from seven MR images," Magnetic Resonance in Medicine, vol. 39, no. 6, pp. 928-934, 1998.

[22] D. Le Bihan, J. F. Mangin, C. Poupon et al., "Diffusion tensor imaging: concepts and applications," Journal of Magnetic Resonance Imaging, vol. 13, no. 4, pp. 534-546, 2001.

[23] P. J. Basser, J. Mattiello, and D. LeBihan, "MR diffusion tensor spectroscopy and imaging," Biophysical Journal, vol. 66, no. 1, pp. 259-267, 1994.

[24] P. J. Basser, "Inferring microstructural features and the physiological state of tissues from diffusion-weighted images," NMR in biomedicine, vol. 8, no. 7-8, pp. 333-344, 1995.

[25] S. Mori and J. Zhang, "Principles of diffusion tensor imaging and its applications to basic neuroscience research," Neuron, vol. 51, no. 5, pp. 527-539, 2006.

[26] S. Mori, B. J. Crain, V. Chacko, and P. van Zijl, "Threedimensional tracking of axonal projections in the brain by magnetic resonance imaging," Annals of Neurology, vol. 45, no. 2, pp. 265-269, 1999.

[27] S. Mori, K. Oishi, H. Jiang et al., "Stereotaxic white matter atlas based on diffusion tensor imaging in an ICBM template," NeuroImage, vol. 40, no. 2, pp. 570-582, 2008.

[28] P. J. Basser, S. Pajevic, C. Pierpaoli, J. Duda, and A. Aldroubi, "In vivo fiber tractography using DT-MRI data," Magnetic Resonance in Medicine, vol. 44, no. 4, pp. 625-632, 2000.

[29] Z. Yu and B. Morrison, "Experimental mild traumatic brain injury induces functional alteration of the developing hippocampus," Journal of Neurophysiology, vol. 103, no. 1, pp. 499510, 2010.

[30] A. C. Bain and D. F. Meaney, "Tissue-level thresholds for axonal damage in an experimental model of central nervous system white matter injury," Journal of Biomechanical Engineering, vol. 122 , no. 6, pp. 615-622, 2000.

[31] M. D. Tang-Schomer, A. R. Patel, P. W. Baas, and D. H. Smith, "Mechanical breaking of microtubules in axons during dynamic stretch injury underlies delayed elasticity, microtubule disassembly, and axon degeneration," FASEB Journal, vol. 24, no. 5, pp. 1401-1410, 2010.

[32] S. S. Fowler, J. P. Leonetti, J. C. Banich, J. M. Lee, R. Wurster, and M. R. I. Young, "Duration of neuronal stretch correlates with functional loss," Otolaryngology-Head and Neck Surgery, vol. 124, no. 6, pp. 641-644, 2001.

[33] H. von Holst, "Organic bioelectrodes in clinical neurosurgery," Biochimica et Biophysica Acta, 2012. 


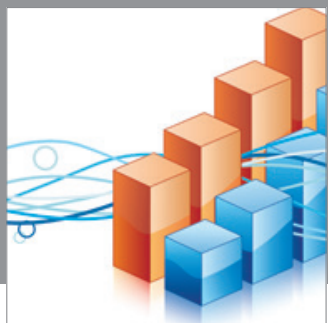

Advances in

Operations Research

mansans

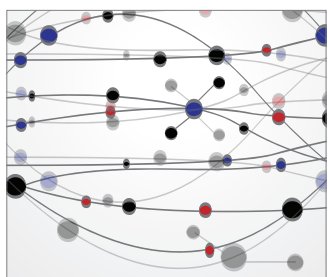

The Scientific World Journal
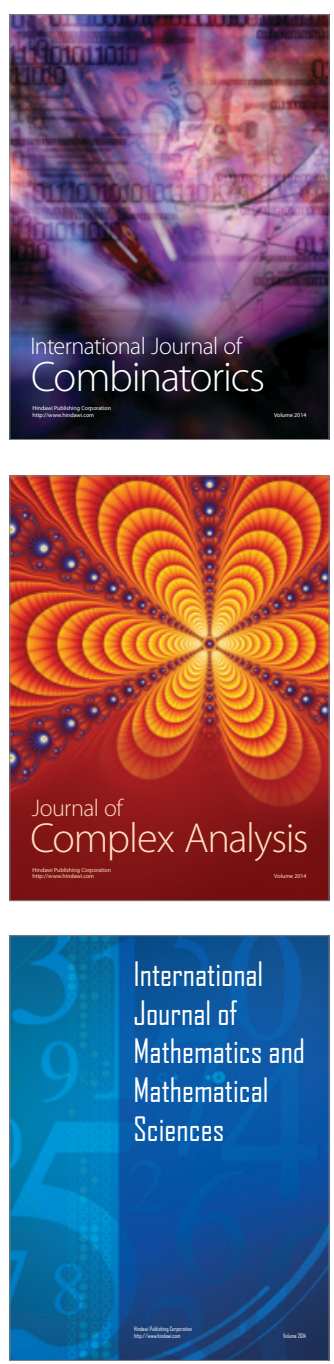
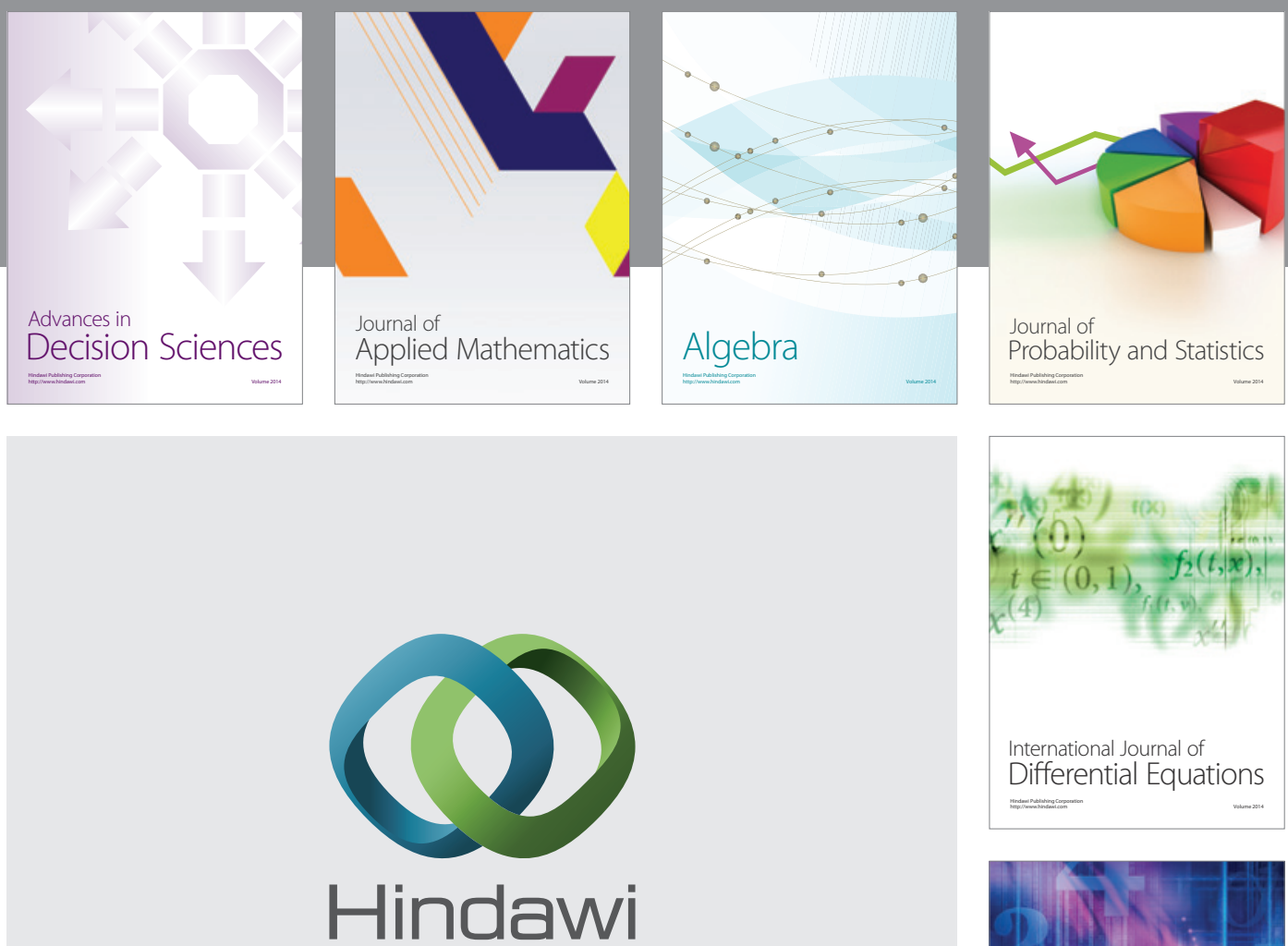

Submit your manuscripts at http://www.hindawi.com
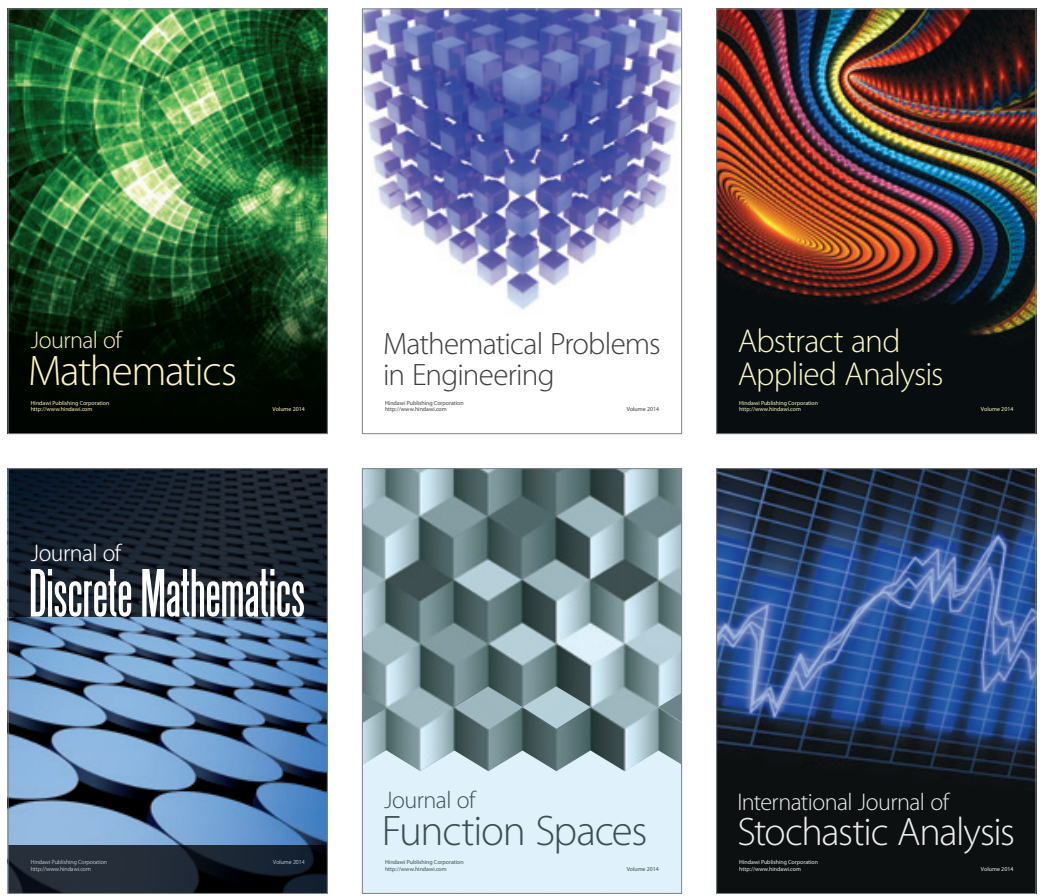

Journal of

Function Spaces

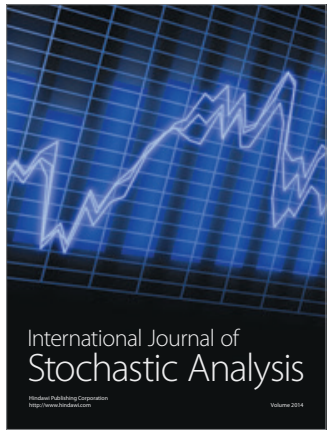

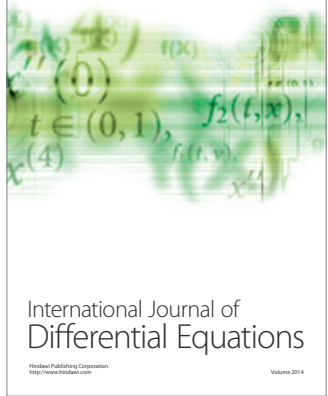
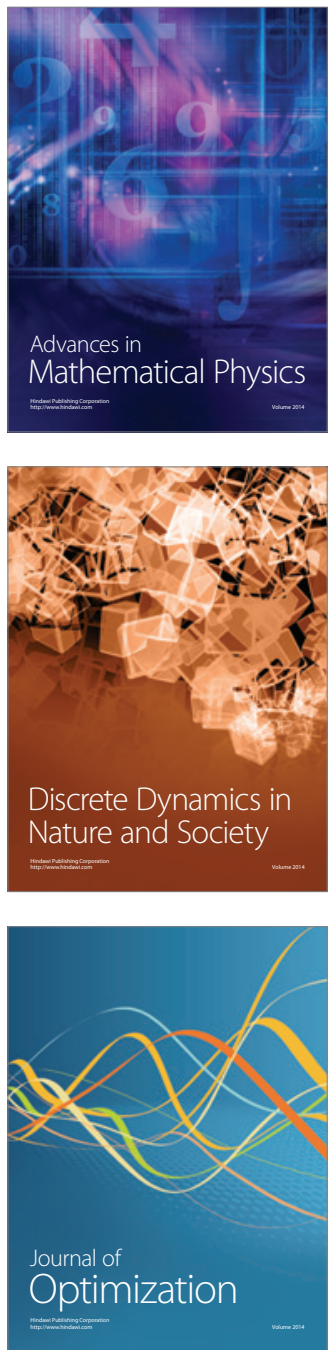